\title{
AMMONIA AS A SOURCE OF GASTRIC HYPOACIDITY IN PATIENTS WITH UREMIA *
}

\author{
By CHARLES S. LIEBER † $\ddagger$ AND ANDRÉ LEFÈVRE \\ (From the Department of Internal Medicine, Brussels University, and the Fondation Reine \\ Elisabeth, Brugmann Hospital, Brussels, Belgium)
}

(Submitted for publication March 3, 1959; accepted March 26, 1959)

Ammonia is produced when urea passes from the blood into gastric secretion, a reaction catalyzed by a urease, present in the gastric mucosa of man and several animals (1-14). Fitzgerald and Murphy (15) have suggested that the ammonia formed in this process may have an important protective function by neutralizing gastric acidity. This concept was based on the observation in man that administration of urea in amounts sufficient to raise temporarily the blood urea level by 12 mg. per cent was followed by a decrease of gastric acidity. Burke and Page (16) were unable to confirm these findings, and Kornberg, Davies and Wood (17-19) presented evidence which suggested that, at least in the cat, the urease found in the stomach is of bacterial origin and does not influence gastric acid secretion.

This paper compares gastric ammonia and acid contents in normal and uremic subjects, the latter known to exhibit an elevated gastric ammonia concentration (20-22). Because of the influence of antibiotics on intestinal ammonia production (23-25), their effects on gastric ammonia and acid concentration were studied.

\section{MATERIALS AND METHODS}

Fifty hospitalized subjects were studied, 24 without evidence of renal dysfunction, 25 with uremia due to chronic renal disease, and one with azotemia due to obstructive uropathy. The blood urea concentration in the patients with uremia varied from 40 to $174 \mathrm{mg}$. nitrogen per cent with an average of $100.5 \mathrm{mg}$. $\mathrm{N}$ per cent. The patients were fasted for 16 hours and gastric juice collected by a Levine tube with the patients placed in the left lateral position, as proposed by Henning (26), to facilitate the quantitative recovery of gastric secretion (27). After a 45 minute period of stabilization, gastric juice was as-

\footnotetext{
* Presented in abstract form at the World Congress of Gastroenterology, Washington, D. C., May, 1958.

$\uparrow$ Supported by a grant from the Fonds National de la Recherche Scientifique Belge.

$\ddagger$ Present address: Thorndike Memorial Laboratory, Boston City Hospital, Boston, Mass.
}

pirated every 15 minutes and collected for examination. Basal secretion was studied using the four specimens collected during the first hour. At the end of this period, $0.005 \mathrm{mg}$. per $\mathrm{Kg}$. histamine dihydrochloride was administered subcutaneously, and the effect of this drug on volume and composition of gastric secretion was determined on the four specimens collected during the second hour. This procedure was performed before and after administration of various antibiotics. 1 Twenty $\mathrm{mg}$. per $\mathrm{Kg}$. oxytetracycline was given orally for four to seven days to five nonuremic and six uremic subjects; $300 \mathrm{mg}$. of the same drug was injected intramuscularly daily for three to eight days to three nonuremics and three uremics. Five uremic and five nonuremic subjects received $20 \mathrm{mg}$. per $\mathrm{Kg}$. chloramphenicol orally for four to seven days and six nonuremic and five uremic subjects were treated similarly with $20 \mathrm{mg}$. per $\mathrm{Kg}$. erythromycin.

Venous blood urea was measured by the urease method (28), gastric juice was analyzed for chlorides (29) and free and total acid (dimethyl-aminoazobenzol and phenolphthalein as indicators). Ammonia and urea were measured (28) in neutralized gastric juice.

\section{RESULTS}

\section{A. Comparison between gastric juice in uremic and nonuremic subjects}

Increased blood urea was associated with high gastric urea and ammonia concentration. This was found both in the basal state and after administration of histamine. In addition, the free and the total acid concentrations were significantly lower $(p<0.05)$ in uremic patients as compared to nonuremic individuals (Table I).

\section{B. Action of antibiotics on gastric ammonia and urea contents}

Mean ammonia concentrations of basal and posthistamine gastric secretions were lowered significantly $(p<0.05)$ in the patients treated by oral oxytetracycline (Table II). In both non-

1 The authors wish to thank the following companies for making these antibiotics available: Lepetit (chloramphenicol), Lilly (erythromycin) and Pfizer (oxytetracycline). 
TABLE I

Comparison between gastric secretion of uremic and nonuremic subjects

\begin{tabular}{|c|c|c|c|c|c|c|c|}
\hline & & $\begin{array}{l}\text { Free } \\
\text { acid }\end{array}$ & $\begin{array}{l}\text { Total } \\
\text { acid }\end{array}$ & Chlorides & $\begin{array}{c}\text { Gastric } \\
\text { ammonia }\end{array}$ & $\begin{array}{c}\text { Gastric } \\
\text { urea }\end{array}$ & Blood urea \\
\hline \multirow{3}{*}{$\begin{array}{l}24 \text { subjects } \\
\text { without } \\
\text { uremia }\end{array}$} & & $m E q . H C l / L$ & $m E q . H C l / L$. & $m E Q . / L$ & mMole/L. & mMole/L. & mMole/L \\
\hline & $\begin{array}{l}\text { Basal secretion } \\
\text { S. D. }\end{array}$ & $\begin{array}{r}22.0 \\
4.5\end{array}$ & $\begin{array}{r}39.6 \\
4.9\end{array}$ & $\begin{array}{r}102.3 \\
5.0\end{array}$ & $\begin{array}{l}5.7 \\
0.9\end{array}$ & $\begin{array}{l}2.8 \\
0.5\end{array}$ & \multirow{2}{*}{$\begin{array}{r}11.4 \pm 0.6 \\
(16.0 \pm 0.8)\end{array}$} \\
\hline & $\begin{array}{l}\text { Posthistamine secretion } \\
\text { S. D. }\end{array}$ & $\begin{array}{r}51.5 \\
7.4\end{array}$ & $\begin{array}{r}65.3 \\
7.6\end{array}$ & $\begin{array}{r}121.9 \\
5.5\end{array}$ & $\begin{array}{l}5.1 \\
0.9\end{array}$ & $\begin{array}{l}2.5 \\
0.5\end{array}$ & \\
\hline \multirow{2}{*}{$\begin{array}{c}26 \text { subjects } \\
\text { with } \\
\text { uremia }\end{array}$} & $\begin{array}{l}\text { Basal secretion } \\
\text { S. D. }\end{array}$ & $\begin{array}{l}8.1 \\
4.1\end{array}$ & $\begin{array}{r}28.2 \\
4.3\end{array}$ & $\begin{array}{r}119.1 \\
5.1\end{array}$ & $\begin{array}{r}37.4 \\
5.2\end{array}$ & $\begin{array}{r}15.9 \\
3.7\end{array}$ & \multirow{2}{*}{$\begin{array}{r}71.8 \pm 4.9 \\
(100.5 \pm 6.9)\end{array}$} \\
\hline & $\begin{array}{l}\text { Posthistamine secretion } \\
\text { S. D. }\end{array}$ & $\begin{array}{r}32.8 \\
6.0\end{array}$ & $\begin{array}{r}55.4 \\
6.6\end{array}$ & $\begin{array}{r}137.7 \\
2.5\end{array}$ & $\begin{array}{r}31.8 \\
5.0\end{array}$ & $\begin{array}{r}17.4 \\
4.0\end{array}$ & \\
\hline
\end{tabular}

uremic and uremic subjects the ammonia concentration was reduced by about 80 per cent of the pretreatment level. An increase of gastric juice urea concentration was associated with the change in ammonia. Both changes were of the same order of magnitude. In five of the six patients with ure- mia receiving oxytetracycline the drop of ammonia was accompanied by a rise of acidity, while in subjects without renal dysfunction no change in acidity was detectable. Similar results were obtained when the values were calculated on the basis of hourly gastric output (Figure 1). No change in

TABLE II

Gastric secretion before (I) and after (II) oral administration of oxytetracycline

\begin{tabular}{|c|c|c|c|c|c|c|c|c|c|c|c|c|c|}
\hline & \multirow{2}{*}{$\begin{array}{c}\text { Case } \\
\text { number }\end{array}$} & \multicolumn{2}{|c|}{ Free acid } & \multicolumn{2}{|c|}{ Total acid } & \multicolumn{2}{|c|}{ Chlorides } & \multicolumn{2}{|c|}{ Ammonia } & \multicolumn{2}{|c|}{ Urea } & \multicolumn{2}{|c|}{ Blood urea } \\
\hline & & I & II & I & II & I & II & I & II & I & II & I & II \\
\hline \multicolumn{2}{|c|}{ Nonuremic subjects } & \multicolumn{2}{|c|}{$m E q . H C l / L$} & \multicolumn{2}{|c|}{$m E q . H C l / L$} & \multicolumn{2}{|c|}{$m E q . / L$} & \multicolumn{2}{|c|}{ mMole/L. } & \multicolumn{2}{|c|}{ mMole/L. } & \multicolumn{2}{|c|}{$\begin{array}{l}\text { \& mMole/L. } \\
\text { (or mg. } N \% \text {. }\end{array}$} \\
\hline $\begin{array}{c}\text { Basal } \\
\text { secretion }\end{array}$ & $\begin{array}{l}1 \\
2 \\
3 \\
4 \\
5\end{array}$ & $\begin{array}{r}32 \\
84 \\
26 \\
0 \\
0\end{array}$ & $\begin{array}{r}2 \\
112 \\
23 \\
0 \\
4\end{array}$ & $\begin{array}{r}62 \\
102 \\
37 \\
10 \\
5\end{array}$ & $\begin{array}{r}23 \\
120 \\
36 \\
15 \\
14\end{array}$ & $\begin{array}{r}81 \\
140 \\
107 \\
94 \\
72\end{array}$ & $\begin{array}{r}97 \\
134 \\
119 \\
112 \\
81\end{array}$ & $\begin{array}{r}11.7 \\
4.0 \\
1.9 \\
11.9 \\
2.7\end{array}$ & $\begin{array}{l}2.0 \\
1.8 \\
1.2 \\
1.0 \\
0.8\end{array}$ & $\begin{array}{l}6.2 \\
0.6 \\
2.4 \\
1.9 \\
3.5\end{array}$ & $\begin{array}{r}11.2 \\
3.6 \\
3.5 \\
10.3 \\
6.6\end{array}$ & $\begin{array}{r}17.4(24.4) \\
10.5(14.7) \\
16.6(23.3) \\
14.0(19.6) \\
8.2(11.5)\end{array}$ & $\begin{array}{l}22.0(30.8) \\
12.8(17.9) \\
11.9(16.7) \\
11.4(16.0)\end{array}$ \\
\hline \multirow{3}{*}{$\begin{array}{l}\text { Posthistamine } \\
\text { secretion }\end{array}$} & Mean & 28.4 & 28.2 & 43.2 & 41.6 & 99 & 109 & 6.4 & 1.4 & 2.9 & 7.0 & $13.3(18.6)$ & $14.5(20.3)$ \\
\hline & $\begin{array}{r}1 \\
3 \\
\\
\\
5\end{array}$ & $\begin{array}{r}89 \\
51 \\
0 \\
31\end{array}$ & $\begin{array}{r}61 \\
66 \\
3 \\
34\end{array}$ & $\begin{array}{r}108 \\
62 \\
10 \\
43\end{array}$ & $\begin{array}{l}78 \\
78 \\
26 \\
44\end{array}$ & $\begin{array}{l}143 \\
116 \\
108 \\
100\end{array}$ & $\begin{array}{l}128 \\
137 \\
119 \\
126\end{array}$ & $\begin{array}{r}7.6 \\
2.2 \\
14.3 \\
2.6\end{array}$ & $\begin{array}{l}1.2 \\
0.7 \\
0.9 \\
0.8\end{array}$ & $\begin{array}{l}8.6 \\
2.1 \\
1.2 \\
2.4\end{array}$ & $\begin{array}{l}7.8 \\
2.9 \\
9.8 \\
4.8\end{array}$ & & \\
\hline & Mean & 43 & 41 & 56 & 57 & 117 & 128 & 6.7 & 0.9 & 3.6 & 6.3 & & \\
\hline Uremic subjects & $\begin{array}{r}6 \\
7 \\
9 \\
10 \\
11\end{array}$ & $\begin{array}{l}0 \\
0 \\
0 \\
0\end{array}$ & $\begin{array}{r}26 \\
0 \\
0 \\
17 \\
10\end{array}$ & $\begin{array}{l}24 \\
10 \\
14 \\
13\end{array}$ & $\begin{array}{l}47 \\
13 \\
34 \\
20\end{array}$ & $\begin{array}{r}127 \\
98 \\
128 \\
122\end{array}$ & $\begin{array}{r}119 \\
96 \\
119\end{array}$ & $\begin{array}{l}79.0 \\
74.0 \\
27.6 \\
61.0 \\
32.9\end{array}$ & $\begin{array}{r}12.0 \\
3.4 \\
14.0 \\
25.6 \\
2.0\end{array}$ & $\begin{array}{l}0.6 \\
0 \\
5.2 \\
2.7 \\
0.3\end{array}$ & $\begin{array}{l}70.0 \\
48.6 \\
45.6 \\
15.1 \\
22.4\end{array}$ & $\begin{array}{rr}110 & (154) \\
103 & (144) \\
52 & (73) \\
75 & (105) \\
54 & (76)\end{array}$ & $\begin{array}{r}124(174) \\
108(151) \\
82(115) \\
73(102) \\
33(46)\end{array}$ \\
\hline $\begin{array}{c}\text { Basal } \\
\text { secretion }\end{array}$ & Mean & $\mathbf{0}$ & 13 & 15 & 29 & 119 & 111 & 54.9 & 11.4 & 1.8 & 40.3 & $78.8(110.3)$ & $84(117.6)$ \\
\hline \multirow[t]{2}{*}{$\begin{array}{l}\text { Posthistamine } \\
\text { secretion }\end{array}$} & $\begin{array}{r}6 \\
7 \\
8 \\
\mathrm{e} \\
9 \\
10 \\
11\end{array}$ & $\begin{array}{l}0.6 \\
67 \\
81 \\
51 \\
45 \\
0\end{array}$ & $\begin{array}{r}44 \\
129 \\
109 \\
87 \\
37 \\
7\end{array}$ & $\begin{array}{l}42 \\
97 \\
98 \\
73 \\
64 \\
11\end{array}$ & $\begin{array}{r}74 \\
138 \\
128 \\
108 \\
60 \\
13\end{array}$ & $\begin{array}{l}136 \\
156 \\
134 \\
139 \\
136 \\
133\end{array}$ & $\begin{array}{r}136 \\
145 \\
145 \\
148 \\
134 \\
84\end{array}$ & $\begin{array}{r}71.0 \\
65.0 \\
3.9 \\
34.7 \\
42.0 \\
30.5\end{array}$ & $\begin{array}{r}10.0 \\
3.0 \\
0.7 \\
14.4 \\
24.8 \\
1.9\end{array}$ & $\begin{array}{r}0.6 \\
0.5 \\
23.2 \\
1.2 \\
0.5 \\
1.5\end{array}$ & $\begin{array}{l}68.0 \\
39.4 \\
35.1 \\
27.1 \\
13.1 \\
23.5\end{array}$ & $52 \cdot(73)$ & $73(102)$ \\
\hline & Mean & 41 & 69 & 64 & 87 & 139 & 132 & 41.2 & 9.1 & 4.6 & 34.3 & & \\
\hline
\end{tabular}


chloride content or volume of gastric juice was observed. Intramuscular administration of oxytetracycline was less effective than when given by mouth, both in reducing gastric ammonia and increasing acidity.

Neither gastric ammonia, acid, nor chloride was significantly influenced by chloramphenicol $(p>$ 0.05) (Table III). Individual responses to erythromycin varied (Table IV); in some subjects gastric ammonia fell considerably, while in others there was no change. The differences were not found to be statistically significant $(p>0.05)$.

The observed antibiotic effect on gastric secretion was reversible. In four patients with uremia whose gastric ammonia had been reduced by antibiotics, it rose again after cessation of the treatment.

C. Relationship between changes in ammonia and acid concentrations in gastric secretion

The relationship between spontaneous changes in gastric ammonia and acid concentration was

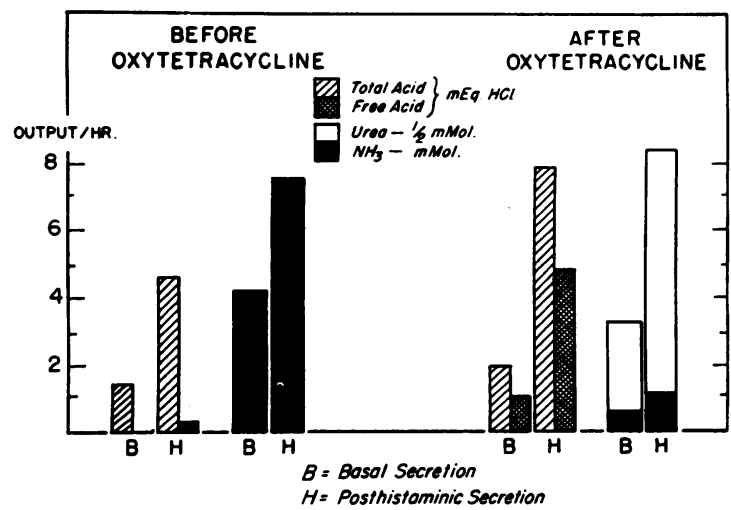

Fig. 1. Gastric Secretion Before and After Seven Days of Oral Oxytetracycline Treatment (20 mg. PER KG. DAILY)

Uremic patient (blood urea, 154 to $174 \mathrm{mg}$. N per cent).

studied in the patient in whom azotemia due to obstructive uropathy was relieved by cystostomy. The gastric ammonia content was lowered proportionally and gastric acidity rose to a similar extent with the fall of blood urea (Figure 2). Ad-

TABLE III

Gastric secretion before (I) and after (II) oral administration of chloramphenicol

\begin{tabular}{|c|c|c|c|c|c|c|c|c|c|c|c|c|c|}
\hline & \multirow{2}{*}{$\begin{array}{c}\text { Case } \\
\text { number }\end{array}$} & \multicolumn{2}{|c|}{ Free acid } & \multicolumn{2}{|c|}{ Total acid } & \multicolumn{2}{|c|}{ Chlorides } & \multicolumn{2}{|c|}{ Ammonia } & \multicolumn{2}{|c|}{ Urea } & \multicolumn{2}{|c|}{ Blood urea } \\
\hline & & I & II & I & II & I & II & I & II & I & II & I & II \\
\hline Nopuremic subie & iects & \multicolumn{2}{|c|}{$m E q . H C l / L$. } & \multicolumn{2}{|c|}{$m E q . H C l / L$} & \multicolumn{2}{|c|}{$m E q . / L$} & \multicolumn{2}{|c|}{ mMole/L. } & \multicolumn{2}{|c|}{ f mMole/L. } & \multicolumn{2}{|c|}{$\begin{array}{l}\frac{1}{m} m M o ! e / L \\
\text { (or mg. } N \% \text { ) }\end{array}$} \\
\hline \multirow[t]{2}{*}{$\begin{array}{l}\text { Basal } \\
\text { secretion }\end{array}$} & $\begin{array}{l}12 \\
13 \\
14 \\
15 \\
16\end{array}$ & $\begin{array}{r}54 \\
21 \\
36 \\
0 \\
0\end{array}$ & $\begin{array}{r}26 \\
16 \\
60 \\
7 \\
0\end{array}$ & $\begin{array}{r}72 \\
46 \\
47 \\
6 \\
11\end{array}$ & $\begin{array}{r}41 \\
22 \\
68 \\
22 \\
4\end{array}$ & $\begin{array}{r}130 \\
126 \\
105 \\
55 \\
72\end{array}$ & $\begin{array}{l}124 \\
124 \\
123 \\
107 \\
146\end{array}$ & $\begin{array}{r}11.1 \\
11.5 \\
3.5 \\
2.6 \\
4.8\end{array}$ & $\begin{array}{l}5.1 \\
5.4 \\
3.1 \\
3.0 \\
0.5\end{array}$ & $\begin{array}{l}0.4 \\
1.5 \\
1.8 \\
3.9 \\
8.9\end{array}$ & $\begin{array}{l}2.3 \\
3.0 \\
0.4 \\
3.3 \\
9.3\end{array}$ & $\begin{array}{l}13.9(19.5) \\
15.4(21.6) \\
10.0(14.0) \\
14.0(19.6) \\
15.9(22.3)\end{array}$ & $\begin{array}{r}12.7(17.8) \\
10.0(14.0) \\
6.0(8.4) \\
10.9(15.3) \\
14.5(20.3)\end{array}$ \\
\hline & Mean & 20 & 22 & 36 & 31 & 97 & 125 & 6.7 & 3.4 & 3.3 & 3.7 & $13.8(19.3)$ & $10.8(15.1)$ \\
\hline \multirow[t]{2}{*}{$\begin{array}{l}\text { Posthistamine } \\
\text { secretion }\end{array}$} & $\begin{array}{l}12 \\
13 \\
14 \\
15 \\
16\end{array}$ & $\begin{array}{r}92 \\
63 \\
61 \\
48 \\
0\end{array}$ & $\begin{array}{r}99 \\
44 \\
75 \\
53 \\
0\end{array}$ & $\begin{array}{r}110 \\
78 \\
70 \\
58 \\
12\end{array}$ & $\begin{array}{r}111 \\
73 \\
91 \\
73 \\
10\end{array}$ & $\begin{array}{r}155 \\
143 \\
128 \\
128 \\
60\end{array}$ & $\begin{array}{l}160 \\
134 \\
143 \\
135 \\
138\end{array}$ & $\begin{array}{l}7.1 \\
9.4 \\
3.1 \\
5.1 \\
3.6\end{array}$ & $\begin{array}{l}3.8 \\
6.3 \\
2.5 \\
2.5 \\
1.8\end{array}$ & $\begin{array}{l}1.2 \\
0.7 \\
1.7 \\
3.0 \\
9.1\end{array}$ & $\begin{array}{r}0.9 \\
2.1 \\
0.4 \\
2.5 \\
10.2\end{array}$ & & \\
\hline & Mean & 53 & 54 & 66 & 72 & 123 & 142 & 5.7 & 3.4 & 3.1 & 3.2 & & \\
\hline \multirow[t]{2}{*}{$\begin{array}{c}\text { Basal } \\
\text { secretion }\end{array}$} & $\begin{array}{l}17 \\
18 \\
19 \\
38 \\
39\end{array}$ & $\begin{array}{l}0 \\
0 \\
6 \\
0 \\
0\end{array}$ & $\begin{array}{r}18 \\
0 \\
35 \\
20 \\
3\end{array}$ & $\begin{array}{r}14 \\
4 \\
22 \\
12 \\
7\end{array}$ & $\begin{array}{l}42 \\
12 \\
56 \\
40 \\
23\end{array}$ & $\begin{array}{l}128 \\
140 \\
107 \\
122 \\
113\end{array}$ & $\begin{array}{l}138 \\
134 \\
158 \\
105 \\
130\end{array}$ & $\begin{array}{r}61.0 \\
47.0 \\
8.6 \\
34.7 \\
23.3\end{array}$ & $\begin{array}{r}52.1 \\
48.0 \\
12.0 \\
3.4 \\
14.2\end{array}$ & $\begin{array}{r}2.7 \\
18.0 \\
20.5 \\
2.2 \\
1.8\end{array}$ & $\begin{array}{r}0.2 \\
20.0 \\
22.6 \\
31.2 \\
4.4\end{array}$ & $\begin{array}{l}75.0(105.0) \\
74.0(104.0) \\
45.5(63.7) \\
48.5(67.9) \\
30.0(42.0)\end{array}$ & $\begin{array}{ll}62.5 & (87.5) \\
81.0 & (113.4) \\
50.0 & (70.0) \\
50.1 & (70.1) \\
28.6 & (40.0)\end{array}$ \\
\hline & Mean & 1.2 & 15.2 & 11.8 & 34.6 & 122 & 133 & 34.9 & 25.9 & 9.0 & 15.7 & $54.6(76.4)$ & $52.4 \quad(73.4)$ \\
\hline \multirow{2}{*}{$\begin{array}{l}\text { Posthistamine } \\
\text { secretion }\end{array}$} & $\begin{array}{l}17 \\
18 \\
19 \\
38\end{array}$ & $\begin{array}{r}45 \\
0 \\
46 \\
0\end{array}$ & $\begin{array}{r}57 \\
0 \\
74 \\
25\end{array}$ & $\begin{array}{r}64 \\
8 \\
66 \\
11\end{array}$ & $\begin{array}{l}78 \\
14 \\
95 \\
38\end{array}$ & $\begin{array}{l}136 \\
150 \\
141 \\
122\end{array}$ & $\begin{array}{l}154 \\
146 \\
160 \\
106\end{array}$ & $\begin{array}{l}42.0 \\
48.0 \\
10.9 \\
34.6\end{array}$ & $\begin{array}{r}45.0 \\
46.0 \\
8.5 \\
3.8\end{array}$ & $\begin{array}{r}0.5 \\
25.0 \\
13.2 \\
3.5\end{array}$ & $\begin{array}{r}0.3 \\
28.0 \\
15.9 \\
35.8\end{array}$ & & \\
\hline & Mean & 22.8 & 39.0 & 37.3 & 56.3 & 137 & 142 & 33.9 & 25.8 & 10.6 & 20.0 & & \\
\hline
\end{tabular}


TABLE IV

Gastric secretion before $(I)$ and after $(I I)$ oral administration of erythromycin

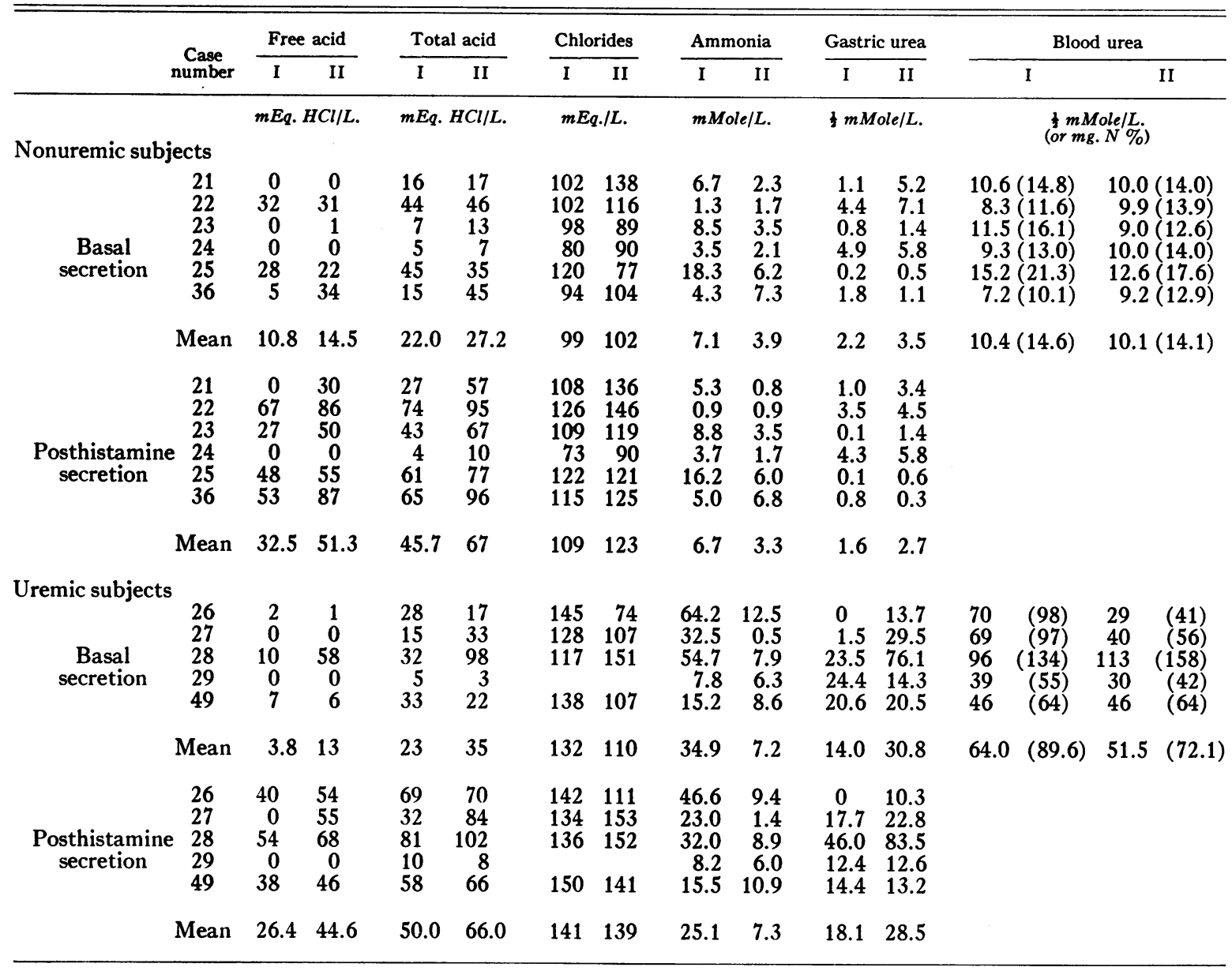

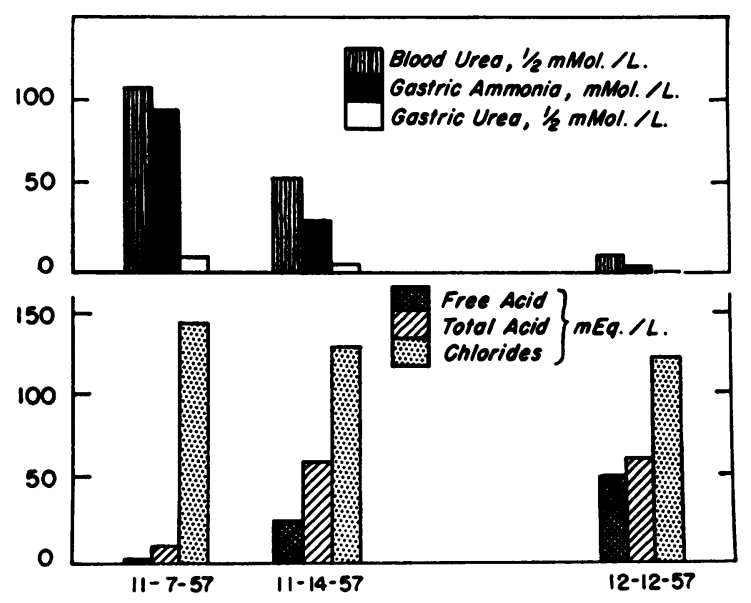

Fig. 2. Effects of a Decrease in Blood Urea on Gastric Ammonia and Acidity (Posthistamine SeCRETION) ministration of antibiotics resulted in a significant drop of gastric ammonia $(\mathrm{p}<0.01)$ and a significant increase of gastric acidity $(\mathrm{p}<0.05)$ in eight patients with uremia due to a chronic glomerulonephritis in whom no change in blood urea occurred during the period of investigation (Table V, Group A). In a similar group (Table V, Group B) of nine patients with uremia, where no marked reduction of gastric ammonia occurred, no change of acidity was observed.

\section{DISCUSSION}

Several authors $(14,15,22,30)$ have shown that the concentration of ammonia in gastric juice is roughly proportional to the blood urea level; this has been confirmed by our results.

Anemia, lowered blood carbon dioxide content, 
TABLE $v$

Seventeen patients with stable uremia: Gastric secretion before and after antibiotic treatment with $(A)$ and without $(B)$ significant reduction in gastric ammonia

\begin{tabular}{|c|c|c|c|c|c|c|c|}
\hline & & \multicolumn{2}{|c|}{ Before antibiotic treatment } & \multicolumn{2}{|c|}{ After antibiotic treatment } & \multicolumn{2}{|c|}{ Difference } \\
\hline & & $\begin{array}{c}\text { Gastric } \\
\text { ammonia }\end{array}$ & $\begin{array}{l}\text { Free } \\
\text { acid }\end{array}$ & $\begin{array}{c}\text { Gastric } \\
\text { ammonia }\end{array}$ & $\begin{array}{l}\text { Free } \\
\text { acid }\end{array}$ & $\begin{array}{c}\text { Gastric } \\
\text { ammonia }\end{array}$ & $\begin{array}{l}\text { Free } \\
\text { acid }\end{array}$ \\
\hline \multirow{3}{*}{$\begin{array}{r}\text { Group A } \\
\text { (8 uremic } \\
\text { patients) }\end{array}$} & & $\begin{array}{c}\text { mMolel } \\
L .\end{array}$ & $\begin{array}{c}m E q_{.} \\
\dot{L} .\end{array}$ & $\begin{array}{c}\text { mMolel } \\
L .\end{array}$ & $\begin{array}{c}m E q . \\
L\end{array}$ & $\begin{array}{l}m M o l e \mid \\
L\end{array}$ & $\begin{array}{c}m E q_{\dot{L}} \\
\mathbf{L}\end{array}$ \\
\hline & $\begin{array}{l}\text { Basal secretion } \\
\text { S. D. }\end{array}$ & $\begin{array}{r}49.8 \\
6.6\end{array}$ & $\begin{array}{l}1.4 \\
1.4\end{array}$ & $\begin{array}{l}7.6 \\
3.4\end{array}$ & $\begin{array}{r}18.7 \\
7.5\end{array}$ & $\begin{array}{r}-42.2 \\
5.2\end{array}$ & $\begin{array}{r}+17.3 \\
6.3\end{array}$ \\
\hline & $\begin{array}{l}\text { Posthistamine secretion } \\
\text { S. D. }\end{array}$ & $\begin{array}{r}41.0 \\
6.1\end{array}$ & $\begin{array}{l}21.6 \\
10.6\end{array}$ & $\begin{array}{l}5.6 \\
1.7\end{array}$ & $\begin{array}{l}52.2 \\
15.0\end{array}$ & $\begin{array}{r}-35.4 \\
6.0\end{array}$ & $\begin{array}{r}+30.6 \\
7.7\end{array}$ \\
\hline \multirow{2}{*}{$\begin{array}{r}\text { Group B } \\
\text { (9 uremic } \\
\text { patients) }\end{array}$} & $\begin{array}{l}\text { Basal secretion } \\
\text { S. D. }\end{array}$ & $\begin{array}{r}26.7 \\
6.9\end{array}$ & $\begin{array}{r}12.8 \\
9.8\end{array}$ & $\begin{array}{r}23.1 \\
6.7\end{array}$ & $\begin{array}{r}12.7 \\
6.7\end{array}$ & $\begin{array}{r}3.6 \\
2.1\end{array}$ & $\begin{array}{r}0.1 \\
12.3\end{array}$ \\
\hline & $\begin{array}{l}\text { Posthistamine secretion } \\
\text { S. D. }\end{array}$ & $\begin{array}{r}24.0 \\
5.9\end{array}$ & $\begin{array}{l}46.2 \\
10.6\end{array}$ & $\begin{array}{r}19.2 \\
5.7\end{array}$ & $\begin{array}{l}47.4 \\
11.6\end{array}$ & $\begin{array}{r}-4.8 \\
2.0\end{array}$ & $+\begin{array}{r}1.2 \\
7.0\end{array}$ \\
\hline
\end{tabular}

disturbances of water and electrolyte balance (31) and gastritis (32) have been suggested previously as causes for gastric hypoacidity in uremia. Although it is possible that these factors may contribute to the encountered low gastric acidity, no experimental proof that they are responsible has been presented.

The hypothesis that gastric ammonia reduces acidity (15) seemed more plausible but has not been supported previously by experimental evidence in uremic patients.

The demonstration that reduction of gastric ammonia after antibiotic treatment increased acidity of patients with uremia and in vitro addition of ammonia decreased acidity (33) clearly relates hypoacidity to ammonia.

In uremic patients, reduction of gastric ammonia causes gastric acidity to rise to a level similar to that of nonuremic subjects. In the basal state, where gastric acid secretion is normally low, the decrease in ammonia may be greater than the increase of acidity. After histamine stimulation, the ammonia decrease parallels the increased acidity.

Administered antibiotics did not influence gastric acidity in the absence of marked changes of anmonia. This was seen in both control subjects and uremic patients in whom antibiotics had little or no effect on gastric ammonia. The encountered differences in ability of oxytetracycline, erythromycin and chloramphenicol to influence gastric ammonia are unexplained; they may be related to the different antibiotic spectra or pharmacodynamic effects. Since bacteria reside in the stomach under physiological conditions $(34,35)$ and urease activity of gastrointestinal organism is reduced by antibiotics (36-39), it seems probable that the ammonia-reducing effect of oxytetracycline and erythromycin is related to their antibiotic properties rather than to a pharmacodynamic effect.

After use of oxytetracycline, an increased incidence of blood in gastric juice was noted and may be attributed to the occurrence of gastric ulcerations after such treatment (40). Theoretically, return of normal acidity coupled with the effects of retained urinary excretory products on mucous membranes may predispose to ulcerations and gastroduodenal bleeding (41).

Effects of therapy on gastric ammonia and acidity may also be of practical importance in patients with liver disease and azotemia who exhibit an elevated gastric ammonia. Reduction of gastric ammonia in patients with cirrhosis can be achieved with oxytetracycline (42) or neomycin (43).

\section{SUM M ARY}

Twenty-six patients with uremia had high gastric ammonia and low free and total acid concentrations, compared with 24 control subjects without evidence of renal dysfunction.

Spontaneous and induced reduction of gastric ammonia in patients with uremia was accompanied by an equivalent increase in gastric acidity, indicating the contribution of ammonia to hypoacidity in uremia. 


\section{ACKNOWLEDGMENTS}

We are indebted to Drs. P. P. Lambert and E. Jacobs (Brussels), C. S. Davidson and R. Schmid (Boston) and C. M. Leevy (New Jersey) for valuable help and advice.

\section{REFERENCES}

1. Berend, N., and Hollan, S. Uber Magen urease. Ber. ges. Physiol. 1941, 126, 478.

2. Cardin, A. L'ureasi nella mucosa gastrica del feto. Arch. Sci. biol. (Bologna) 1933, 19, 76.

3. Glick, D., Zak, E., and Von Korff, R. Role of urease in the gastric mucosa. Amer. J. Physiol. 1950, $163,386$.

4. Linderstr $\varnothing \mathrm{m}-\mathrm{Lang}, \mathrm{K}$, , and Ohlsen, A. S. Distribution of urease in dog's stomach. Enzymologia 1936-37, 1, 92.

5. Luck, J. M., and Seth, T. N. The physiology of gastric urease. Biochem. J. 1925, 19, 357.

6. Martin, L. Studies on an urea-splitting enzyme found in the gastric juice. Bull. Johns Hopk. Hosp. 1933, $52,166$.

7. Martin, L. Protein nitrogen and nonprotein nitrogen determinations on gastric juice. J. Amer. med. Ass. 1933, 100, 1475.

8. Rigoni, M. La presenza di ureasi nella mucosa gastrica umana. Boll. Soc. ital. Biol. sper. 1929, 4, 1166.

9. Sumida, S. Durchblutungsversuche des Magens. IV. Mitteilung über Durchblutungsversuche des Magens mit Harnstoff. J. Biochem. (Tokyo) 1934, 19, 449.

10. Sumida, S. Durchblutungsversuche des Magens. XII. Mitteilung über die Urease in der Magenwand. J. Biochem. (Tokyo) 1935, 21, 301.

11. Strehler, E. Nierenkrankheiten und Ammoniakgehalt im Blut. Schweiz. med. Wschr. 1943, 1492.

12. Strehler, E. Ubersicht über die Urease und die Bedeutung ihres Vorkommens im Magen. Helv. med. Acta 1955, 1, 83.

13. Von Korff, R. W., and Glick, D. Role of urease in the gastric mucosa. II. In vitro studies with isotopic urea on frog mucosa. Amer. J. Physiol. 1951, 165, 688 .

14. Von Korff, R. W., Ferguson, D. J., and Glick, D. Role of urease in gastric mucosa. III. Plasma urea as source of ammonium ion in gastric juice of histamine-stimulated dog. Amer. J. Physiol. 1951, $165,695$.

15. Fitzgerald, O., and Murphy, P. Studies on the physiological chemistry and clinical significance of urease and urea with special reference to the stomach. Irish J. med. Sci. 1950, 6, 97.

16. Burke, J. O., and Page, S. G. The effects of orally administered urea on healthy subjects and their gastric secretory response to histamine. Gastroenterology 1954, 26, 503.

17. Kornberg, H. L., Davies, R. E., and Wood, D. R. The breakdown of urea in cats not secreting gastric juice. Biochem. J. 1954, 56, 355.
18. Kornberg, H. L., Davies, R. E., and Wood, D. R. Activity and function of gastric urease in the cat. Biochem. J. 1954, 56, 363.

19. Kornberg, H. L., and Davies, R. E. Gastric urease. Physiol. Rev. 1955, 35, 169.

20. Fossel, M. Die Urease der menschlichen Magenschleimhaut. Wien. klin. Wschr. 1948, 60, 60.

21. Hessel, G., Pekelis, E., and Meltzer, H. Die Magensaftsekretion bei nephrektomierten Tieren. Z. ges. exp. Med. 1933, 91, 274.

22. Simici, D., Vladesco, R., and Popesco, M. Recherches sur l'urée et l'ammoniac des liquides gastriques a l'état normal et sur l'élimination de l'urée par l'estomac. C. R. Soc. Biol. (Paris) 1929, 101, 199.

23. Martini, G. A., Phear, E. A., Ruebner, B., and Sherlock, S. The bacterial content of the small intestine in normal and cirrhotic subjects: Relation to methionine toxicity. Clin. Sci. 1957, 16, 35.

24. Phear, E. A., and Ruebner, B. The in vitro production of ammonium and amines by intestinal bacteria in relation to nitrogen toxicity as a factor in hepatic coma. Brit. J. exp. Path. 1956, 37, 253.

25. Silen, W., Harper, H. A., Mawdsley, D. L., and Weirich, W. L. Effect of antibacterial agents on ammonia production within the intestine. Proc. Soc. exp. Biol. (N. Y.) 1955, 88, 138.

26. Henning, N. Uber die Resorption der normalen und entzündeten Magenschleimhaut. Dtsch. Arch. klin. Med. 1930, 166, 205.

27. Kimbel, K. H., and Kinzlmeier, H. Quantitative intragastrale Bestimmung der Säureproduktion des gesunden und kranken Magens. Gastroenterologia 1954, 81, 193.

28. Conway, E. J. Microdiffusion Analysis and Volumetric Error. London, Crosby, Lockwood \& Son, Ltd., 1957.

29. Schales, O., and Schales, S. S. A simple and accurate method for the determination of chloride in biological fluids. J. biol. Chem. 1941, 140, 879.

30. Bessman, S. P., and Staufer, J. C. Factor affecting the appearance of ammonia in the gastric juice (abstract). J. clin. Invest. 1957, 36, 874.

31. Jacobs, E. Retentissement digestif de l'insuffisance rénale. Acta gastro-ent. belg. 1957, 20, 349.

32. Cheli, R., and Dodero, M. Etude biopsique et sécrétoire de la muqueuse gastrique au cours de l'urémie chronique. Acta gastro-ent. belg. 1958, $21,193$.

33. Lieber, C. S. Unpublished data.

34. Henning, N. Die Bakterienbesiedlung des gesunden und kranken Magens. Arch. Verdau.-kr. 1930, 47, 1.

35. Nichols, A. C., and Glenn, P. M. Intubation studies of the human small intestine. XVI. The bacterial flora of the ileum compared with that of throat and stomach in normal subjects. J. Lab. clin. Med. 1939-40, 25, 388. 
36. Walser, M., and Bodenlos, L. J. Urea breakdown in normal man (abstract). J. clin. Invest. 1957, 36, 933.

37. Webster, L. T., Jr., and Davidson, C. S. Sources of blood ammonium after feeding protein to patients with hepatic cirrhosis (abstract). J. clin. Invest. 1956, 35, 742.

38. Chao, F.-C., and Tarver, H. Breakdown of urea in the rat. Proc. Soc. exp. Biol. (N. Y.) 1953, 84, 406.

39. Dintzis, R. Z., and Hastings, A. B. The effect of antibiotics on urea breakdown in mice. Proc. nat. Acad. Sci. (Wash.) 1953, 39, 571.

40. Lieber, C. S., and Desneux, J. J. A propos de l'action ulcérogène gastrique de l'oxytétracycline chez l'homme. Acta gastro-ent. belg. 1957, 20, 738.
41. Verbanck, M., Toussaint, C., Lieber, C. S., and Lefèvre, A. Hémorragies gastro-duodénales compliquant les anuries aiguës au cours du traitement par le rein artificiel. Acta gastro-ent. belg. 1957, 20, 798.

42. Lieber, C. S., and Lefèvre, A. Métabolisme ammoniacal et intermédiaire au cours du coma hépatique. Valeur du dosage de l'ammoniaque sanguin dans l'établissement du diagnostic et la conduite du traitement au cours de la cirrhose. Acta clin. belg. 1958, 13, 328.

43. Webster, L. T., Jr., and Gabuzda, G. J. Relation of azotemia to blood "ammonium" in patients with hepatic cirrhosis. Arch. intern. Med. 1959, 103, 15.

\section{SPECIAL NOTICE TO SUBSCRIBERS}

Post Offices will no longer forward the Journal when you move.

Please notify The Journal of Clinical Investigation, Business

Office, 333 Cedar Street, New Haven 11, Conn., at once when you have a change of address, and do not omit the zone number if there is one. 